\title{
La calidad del servicio interno en el sector hotelero: objeto e instrumento de estudio multidisciplinar
}

Recibido: 17/11/16 · Aceptado: 21/02/17

\author{
Aralí Larios Calderón* \\ Universidad Tecnológica de la Riviera Maya \\ Alfonso González Damián \\ Universidad de Quintana Roo
}

\section{Resumen}

El objetivo de este trabajo es mostrar el estado que presenta en la literatura académica el concepto de calidad del servicio interno en el sector hotelero. Como resultado de la revisión, integración y análisis de artículos científicos, se encontró que el concepto ha sido tomado como objeto e instrumento de estudio por diversas disciplinas, como la económica-administrativa, el marketing, la psicología social y la responsabilidad social empresarial, lo que ha contribuido a una mejor comprensión del concepto. Las investigaciones enfocadas en la calidad del servicio interno aplicadas al sector hotelero son escasas, no obstante, las existentes evidencian la necesidad de desarrollar estudios cualitativos que aborden con profundidad la exploración de factores que inciden en el comportamiento del trabajador para el desarrollo de la calidad del servicio interno.

Palabras clave: Calidad del servicio interno, conceptualización, hotelería, revisión de literatura, servicio interno.

*Correo electrónico: arali_larios@hotmail.com 


\title{
Internal service quality in the hotel sector: an object and instrument of multidisciplinar studies
}

Recieved: 17/11/2016 · Accepted: 21/02/2017

\author{
Aralí Larios Calderón* \\ Universidad Tecnológica de la Riviera Maya \\ Alfonso González Damián \\ Universidad de Quintana Roo
}

\begin{abstract}
The aim of this study is to identify the state of the art in academic literature of the concept of internal service quality in the hotel sector. As the result of the review, integration and analysis of scientific articles it was found that the concept has been adopted as an object and instrument of study for various disciplines, such as economic-administrative, marketing, social psychology and entrepreneurial social responsibility, contributing to a better understanding of the concept. Research focused on the internal service quality applied in the hotel sector has been scarce but nevertheless the few existing studies reflect the need to develop qualitative indepth studies that address the factors that impinge on employee behavior for the development of quality in the internal service.
\end{abstract}

KEY woRDs: Internal service quality, conceptualization, hotel industry, literature review, internal service.

*E-mail: arali_larios@hotmail.com 


\section{Introducción}

En una notoria paradoja, muchas empresas de servicios en sitios de destino turístico internacional, como el caso de Cancún y la Riviera Maya en México, destinan una importante inversión al fortalecimiento de la calidad de sus servicios al cliente, pero no lo hacen en igual proporción para mejorar el servicio que los empleados se proveen permanentemente unos a otros en sus distintos niveles y áreas. Parecería que las organizaciones tienen muy claro que en la medida en que el consumidor turístico vea cumplidas sus expectativas, reportará una mayor satisfacción, lo cual incidirá de forma positiva en sus intenciones de regresar al destino y sus empresas, así como de recomendarlas entre sus conocidos; sin embargo, no han conseguido entender que el consumidor de servicios, y particularmente el de los servicios turísticos, se involucra de manera muy cercana en la producción del servicio y entra en relación directa con los empleados, quienes a su vez, si no reciben información y medios en el momento y en la calidad requeridos, estarán poco preparados y hasta imposibilitados para colaborar con el cliente.

Simple y llanamente, si los empleados no están satisfechos como receptores de servicios de otros empleados y de la organización en general, esto se traducirá también en insatisfacción del cliente externo, con lo que ello implica para la rentabilidad, productividad y competitividad del negocio. Esta es la noción central que subyace en el concepto de calidad del servicio interno; empero, en el camino de realizar estudios aplicados al caso del sector hotelero en la Riviera Maya, se encontró que el propio concepto sigue estando en debate en el ámbito académico y que existen distintas perspectivas que lo abordan, cada una con intencionalidades, marcos teóricos y metodológicos, así como estrategias, técnicas y herramientas variadas. Esta diversidad, que en principio podría ser vista como riqueza conceptual, genera cierta confusión y en algunos estudios incluso abordajes incompletos o sesgados.

Dada esta situación, se planteó como objetivo para el presente documento mostrar el estado actual del concepto de calidad del servicio interno en el sector hotelero en la literatura académica, a través de la revisión, análisis y organización de los estudios publicados en artículos de revistas académicas indizadas, tanto en inglés como en español, especialmente en lo producido en los últimos 20 años. 
La calidad del servicio interno en el sector hotelero: objeto e instrumento de estudio multidisciplinar

\section{Antecedentes}

Se pueden ver esbozos de la conceptualización de la calidad del servicio interno desde la década de 1930 en la etapa del enfoque humanístico de la administración, en donde la esencia de esta disciplina son las personas. Pero es en 1994 cuando aparece el término de calidad del servicio interno en el modelo organizacional denominado cadena de servicio-beneficio desarrollado por Heskett, Jones, Loveman, Sasser y Schlesinger. En ese entonces, el concepto era definido como el ambiente generado a través de los sentimientos del trabajador hacia su trabajo, sus colegas y la empresa. Desde esta corriente se produjo una serie de investigaciones para comprobar la relación entre las variables propuestas por los autores, con la finalidad de que se reconociesen las formas en las que podían influir en la generación de mayores ventas y utilidades para la empresa.

Aunado a lo anterior, hay quienes toman a este concepto a partir de la perspectiva del marketing desde 1995, con la definición de servicio interno de Stauss, originando la preocupación por las necesidades del cliente interno, llevando a estudios sobre la calidad de los servicios al interior de la empresa.

Es un hecho que, en cualquiera de las perspectivas anteriores, el enfoque de investigación es la satisfacción del trabajador, desglosando conceptos en la búsqueda de su comprensión, como el de calidad del servicio interno.

En 1996, Hallowell, Schlesinger y Zornitsky definen la calidad del servicio interno para efectos de su investigación como "la satisfacción del trabajador con los servicios recibidos de provedores del servicio interno" (p. 21), y en 2001, Frost y Kumar utilizan una conceptualización más amplia fundada en la literatura del marketing interno y la calidad del servicio, la cual es usada hoy en día al referirse a este concepto como un ambiente interno basado en el apoyo consciente entre empleados, en donde los clientes son los trabajadores, y el staff de apoyo, que incluye a la gerencia y otros apoyos, son los proveedores del servicio.

Como se puede observar, el concepto se ha convertido en objeto de investigación en las pasadas dos décadas (Homburg y Stock, 2005), derivado de la importancia de la satisfacción del empleado dentro las empresas (Heskett, Jones, Loveman, Sasser y Schlesinger, 1994; Nazeer, Zahid y Azeem, 2014), ya que existen estudios que comprueban que las organizaciones que proveen calidad a 116 los clientes internos son en general exitosas, y que la satisfacción del empleado 
se encadena con el desempeño de la organización (Pantouvakis, 2011), por lo que la mayoría de las investigaciones han sido realizadas con el objetivo pragmático de generar estrategias basadas en la determinación de necesidades del personal, para mantenerlo, desarrollarlo y con ello mejorar la calidad del servicio que impacte en la satisfacción (Mehrparvar, Shahin y Shirouyehzad, 2012).

En este último punto cabe señalar que los estudios han mostrado que la relevancia de abordar este concepto radica en que la calidad del servicio al cliente externo es altamente dependiente de la calidad del servicio interno (Gunawardane, 2009). Sin embargo, hay quienes, como Jun y Cai (2010), ven a la calidad del servicio interno más allá de lograr clientes satisfechos, enfatizando que los resultados de trabajar en la calidad al interior de la empresa pueden producir intercambios internos eficientes entre varios miembros y departamentos de la organización, bajos desperdicios y costos, y un servicio de calidad al cliente externo.

Asimismo, en congruencia con la evolución del concepto de empresa, que ha transitado en el ámbito académico de aquel que establecía que la única responsabilidad de las empresas era generar riqueza para los inversionistas (Friedman, 1970), al más actual y cada vez más ampliamente aceptado de que las empresas son responsables de las consecuencias de su actuar, desde luego en la esfera económica, pero también en la medioambiental y la sociocultural (Hart, 1997), y que con motivo de tal responsabilidad no solo deben rendir cuentas a los inversionistas -shareholders- sino a todas las partes interesadas -stakeholders-, tanto a su interior como en su entorno local y general (Freeman, Wicks y Parmar, 2004).

Esta visión de rendición de cuentas a las partes interesadas considera como una de ellas y singularmente relevante la de los empleados de la empresa, quienes están naturalmente interesados en el éxito económico-financiero de la misma, pero además tienen un alto interés centrado en su propia satisfacción como trabajadores y en la atención a sus necesidades, las de su familia y las de la comunidad en la que residen (Aguilera, Rupp, Williams y Ganapathi, 2007). Esta condición se ha destacado de manera importante en el caso de las empresas hoteleras y en general del sector turismo (Kasim, 2006), que atienden a consumidores en tránsito y que no necesariamente pueden ser considerados como clientes externos en la noción convencional, en tanto que los empleados permanecen y viven constantemente los efectos de la acción empresarial. 
La calidad del servicio interno en el sector hotelero: objeto e instrumento de estudio multidisciplinar

La noción ampliada de empresa ha hecho necesario y ha conducido inevitablemente a una expansión del concepto de calidad del servicio interno, el cual ya no podría encontrar justificación solo en la búsqueda de la competitividad de la empresa, sino también en otros valores como la justicia, el desarrollo sostenible, el empoderamiento, la calidad de vida, etc. Ello implica asimismo la necesidad de revisar lo producido conceptualmente en la búsqueda de resignificarlo, reorganizarlo y replantearlo en perspectivas multidisciplinares y que trasciendan la noción puramente económica.

\section{Metodología}

La presente investigación se basó en la revisión de artículos publicados en journals, con resultados de investigación seleccionados a partir de búsquedas reiteradas centradas en el concepto de calidad del servicio interno y el sector hotelero.

En una primera etapa, la búsqueda de la información se efectuó en las bases de datos especializadas de Elsevier, EBSCOhost y Emerald, utilizando como palabra descriptora "internal service quality", y en español, "calidad del servicio interno”. Se detectó que la búsqueda arrojaba muy pocos artículos que contenían la palabra en el título, palabras clave o en el abstract, por lo que fue necesario realizar revisiones detalladas partiendo de la bibliografía citada en los pocos artículos encontrados inicialmente, para identificar sus referentes disciplinares y teóricos, con lo que se accedió de nuevo a las bases de datos mencionadas, así como a las siguientes: Redalyc (Red de Revistas Científicas de América Latina y el Caribe), Dialnet y Scielo (Scientific Electronic Library Online), y se amplió la búsqueda a lo que mostrara también Google Académico.

Esta búsqueda permitió comprender mejor el tema e identificar las relaciones que hacían en los artículos de calidad del servicio interno con otras variables propuestas por diferentes disciplinas o enfoques. Con base en la información encontrada, se generaron las primeras ideas de agrupación, por lo que se decidió organizar los artículos recolectados de forma digital en dos grupos: uno con los documentos de la primera búsqueda, que significaban los artículos que indagaban en la exploración y medición del concepto, y otro conformado a su vez por cuatro subgrupos, que surgieron de la propia búsqueda, es decir, con un procedimiento heurístico que permitió organizar los artículos de la 
segunda búsqueda, los cuales se apoyaban en distintas disciplinas explicativas del concepto.

A partir de esta organización disciplinar, se analizó la información con la intención de encontrar agrupaciones lógicas entre ella, esto es, en un sentido lógico inductivo, se identificaron dos grupos que pueden ser considerados como perspectivas congruentes con la noción de empresa que subyace en los estudios. Llegados a este punto, se juzgó viable entregar los resultados que se muestran en el presente artículo.

En el ámbito instrumental, se utilizó la aplicación Mendeley, gestor de referencias bibliográficas que facilitó el almacenamiento y organización digital de los documentos, lo cual a su vez incidió en la aceleración de búsquedas en los textos recolectados.

\section{Resultados y discusión}

\section{Estudios de la calidad en el servicio interno a partir de sus definiciones y medición}

En una primera organización de la literatura, se identificaron los estudios que abordan el concepto de calidad del servicio interno y que buscan precisar sus elementos, dimensiones o componentes, con la finalidad de comprender más a fondo su definición y operacionalización conceptual. El cuadro 1 muestra la lista de los resultados desglosada en: autor del artículo, campo de estudio y objetivo de la investigación, además de los elementos, dimensiones o componentes que se encontraron en el estudio.

Cuadro 1. Estudios definiendo la calidad del servicio interno

\begin{tabular}{ccll}
\hline Autor & $\begin{array}{c}\text { Campo } \\
\text { de estudio }\end{array}$ & \multicolumn{1}{c}{$\begin{array}{c}\text { Objetivo } \\
\text { Boshoff y Mels (1995) }\end{array}$} & $\begin{array}{l}\text { Vlementos, dimensio- } \\
\text { nes o componentes } \\
\text { encontrados }\end{array}$ \\
& seguros & $\begin{array}{l}\text { Identificar la influencia } \\
\text { de la supervisión en } \\
\text { la calidad del servicio } \\
\text { interno. }\end{array}$ & $\begin{array}{l}\text { Organizational com- } \\
\text { mitment, supervision, } \\
\text { role stress. }\end{array}$ \\
& &
\end{tabular}


La calidad del servicio interno en el sector hotelero: objeto e instrumento de estudio multidisciplinar

\section{Cuadro 1. Estudios definiendo la calidad del servicio interno (continuación)}

\begin{tabular}{|c|c|c|c|}
\hline Autor & $\begin{array}{c}\text { Campo } \\
\text { de estudio }\end{array}$ & Objetivo & $\begin{array}{l}\text { Elementos, dimensio- } \\
\text { nes o componentes } \\
\text { encontrados }\end{array}$ \\
\hline $\begin{array}{l}\text { Hallowell, Schlesinger } \\
\text { y Zornitsky (1996) }\end{array}$ & $\begin{array}{l}\text { Compañía de } \\
\text { seguros }\end{array}$ & $\begin{array}{l}\text { Identificar si los ele- } \\
\text { mentos de la calidad del } \\
\text { servicio interno afectan } \\
\text { la satisfacción del cliente. }\end{array}$ & $\begin{array}{l}\text { Tools, policies and } \\
\text { procedures, teamwork, } \\
\text { management support, } \\
\text { goal alignment, effec- } \\
\text { tive training, commu- } \\
\text { nication, and rewards } \\
\text { and recognition. }\end{array}$ \\
\hline $\begin{array}{l}\text { Finn, Baker, Marshall } \\
\text { y Anderson (1996) }\end{array}$ & $\begin{array}{l}\text { Departamento de } \\
\text { Compras corpo- } \\
\text { rativo de la firma } \\
\text { farmacéutica } \\
\text { Alcon Laborato- } \\
\text { ries }\end{array}$ & $\begin{array}{l}\text { 1. Identificar los requeri- } \\
\text { mientos de servicio por } \\
\text { parte del cliente interno. } \\
\text { 2. Evaluar la calidad del } \\
\text { servicio interno. }\end{array}$ & $\begin{array}{l}\text { Tender loving care, } \\
\text { Delivering value, Order } \\
\text { processing, Vendor } \\
\text { management, Knowl- } \\
\text { edge, No surprises. }\end{array}$ \\
\hline Jun y Cai (2010) & $\begin{array}{l}\text { Departamento de } \\
\text { Compras }\end{array}$ & $\begin{array}{l}\text { Identificar si los clientes } \\
\text { internos perciben la } \\
\text { calidad del servicio } \\
\text { significativa y positiva- } \\
\text { mente relacionada con su } \\
\text { satisfacción. }\end{array}$ & $\begin{array}{l}\text { Customer intimacy, } \\
\text { Team-based con- } \\
\text { tinuous improvement, } \\
\text { Communication, } \\
\text { Reliability/competence, } \\
\text { Requisition process, } \\
\text { Tangible. }\end{array}$ \\
\hline $\begin{array}{l}\text { Vanniarajan y Sub- } \\
\text { bash (2011) }\end{array}$ & $\begin{array}{l}\text { Servicio financiero } \\
\text { (bancos) }\end{array}$ & $\begin{array}{l}\text { Identificar los aspectos } \\
\text { importantes de la calidad } \\
\text { del servicio interno. }\end{array}$ & $\begin{array}{l}\text { Employees' and } \\
\text { customers' orientation, } \\
\text { team orientation and } \\
\text { employee orientation, } \\
\text { learning environment } \\
\text { and outcome orienta- } \\
\text { tion. }\end{array}$ \\
\hline Chen (2013) & $\begin{array}{l}\text { Industria hotelera } \\
\text { internacional }\end{array}$ & $\begin{array}{l}\text { Construir un modelo } \\
\text { de las influencias sobre } \\
\text { la calidad del servicio } \\
\text { interno. }\end{array}$ & $\begin{array}{l}\text { Bureaucratic organi- } \\
\text { zational culture and } \\
\text { leadership. }\end{array}$ \\
\hline
\end{tabular}


Cuadro 1. Estudios definiendo la calidad del servicio interno (finaliza)

\begin{tabular}{|c|c|c|c|}
\hline Autor & $\begin{array}{c}\text { Campo } \\
\text { de estudio }\end{array}$ & Objetivo & $\begin{array}{c}\text { Elementos, dimensio- } \\
\text { nes o componentes } \\
\text { encontrados }\end{array}$ \\
\hline $\begin{array}{l}\text { Umamaheswari } \\
\text { (2014) }\end{array}$ & $\begin{array}{l}\text { Industria manu- } \\
\text { facturera }\end{array}$ & $\begin{array}{l}\text { 1. Examinar las dimen- } \\
\text { siones de la calidad del } \\
\text { servicio interno. } \\
\text { 2. Identificar los factores } \\
\text { que afectan la calidad del } \\
\text { servicio interno. }\end{array}$ & $\begin{array}{l}\text { 1. Individual, Depart- } \\
\text { mental \& organiza- } \\
\text { tional dimensions. } \\
\text { 2. Roles and re- } \\
\text { sponsibility, service } \\
\text { competency, communi- } \\
\text { cation, resolution and } \\
\text { alignment factors. }\end{array}$ \\
\hline $\begin{array}{l}\text { Sharma, Chuen y } \\
\text { Kingshott (2016) }\end{array}$ & $\begin{array}{l}\text { Industria manu- } \\
\text { facturera }\end{array}$ & $\begin{array}{l}\text { La calidad del servicio } \\
\text { interno tiene un efecto } \\
\text { positivo sobre la satis- } \\
\text { facción del empleado, } \\
\text { compromiso y bienestar } \\
\text { del trabajador. }\end{array}$ & $\begin{array}{l}\text { Employee satisfaction. } \\
\text { Employee commit- } \\
\text { ment. } \\
\text { Employee well-being. }\end{array}$ \\
\hline $\begin{array}{l}\text { Fadil, Singh y Joseph } \\
\text { (2016) }\end{array}$ & $\begin{array}{l}\text { Oficina de ser- } \\
\text { vicios públicos } \\
\text { (gobierno) }\end{array}$ & $\begin{array}{l}\text { Comprender mejor la } \\
\text { influencia de la innova- } \\
\text { ción organizacional en } \\
\text { la calidad del servicio } \\
\text { interno. }\end{array}$ & $\begin{array}{l}\text { Innovation organi- } \\
\text { zational (Innovation } \\
\text { leadership, managerial } \\
\text { levers and business } \\
\text { process). }\end{array}$ \\
\hline
\end{tabular}

Nota: Para efectos de mantener el concepto en inglés de las palabras, no se hizo la traducción de las dimensiones, elementos o componentes.

Fuente: Elaboración propia.

Como se observa en el cuadro 1, de los estudios enfocados a comprender el concepto de calidad del servicio interno solo uno se presenta en el campo del turismo, específicamente en el sector de la industria hotelera. Con el examen de los documentos se identificó que la definición de calidad del servicio interno puede considerarse compuesta por dos términos: servicio interno y calidad. El primero es definido por Stauss (1995 cit. en Large y König, 2009) como los servicios proveídos por distintas unidades organizacionales, o la gente trabajando en departamentos para otras unidades o empleados dentro de la organización; y el segundo está tomado del modelo teórico de Grönroos (1982), que contempla dos dimensiones: la técnica y la funcional, siendo esta última donde se observan aspectos intangibles como las relaciones internas. 
La calidad del servicio interno en el sector hotelero: objeto e instrumento de estudio multidisciplinar

Cabe enfatizar que el principio básico del concepto de servicio al cliente interno es que cada departamento (o a veces trabajadores) recibe trabajo de y/o desarrolla trabajo para otro departamento (otro trabajador). El departamento o empleado que desarrolla el trabajo es el productor del servicio interno y el departamento o empleado que recibe es el cliente interno; su interacción es el encuentro del servicio interno (Gunawardane, 2009). Por lo que la presencia del concepto de calidad del servicio interno está en el encuentro que tienen los departamentos o el recurso humano de la organización en el desempeño del servicio que entre ambos se proveen.

El concepto de calidad del servicio interno ha sido considerado un componente básico para la mayoría de las organizaciones (Hallowell, Schlesinger y Zornitsky, 1996), y ha llegado a convertirse en un elemento de la cultura organizacional de las empresas, por ejemplo las encuestas de la calidad del servicio interno que se aplican como parte de las herramientas culturales de los servicios financieros de la compañía (Cook, 2004), y desde la perspectiva del factor humano, se le ha visto como una fuente potencial de ventaja competitiva sustentable (Pfau et al., 1991 y Albrecht, 1993 cit. en Vanniarajan y Subbash, 2011).

Sin embargo, según mencionan Seibert y Lingle (2007), el avance de este tema en la práctica no ha sido muy significativo, ya que en 1993 se mostraba solo $32 \%$ favorable de satisfacción del cliente interno, y una encuesta de comparación en 2006 de 1266 miembros de la Sociedad Americana de Calidad exhibe únicamente $48 \%$ de satisfacción.

Uno de los aspectos que se identificó en la investigación del estado del conocimiento del concepto de calidad del servicio interno es que para constatar la importancia de la satisfacción del cliente interno, o para comprender los factores que lo conforman, se han aplicado instrumentos para evaluarlo, la mayoría de los cuales utiliza la herramienta SERVQUAL de Parasuraman, Zeithaml y Berry (1988) en investigaciones cuantitativas, y en algunos casos se ha incorporado alguna herramienta cualitativa al estudio como el focus groups o las entrevistas. En este punto es importante señalar que el SERVQUAL es un instrumento que fue diseñado para medir la calidad del servicio al cliente externo, y no al cliente interno, pero su fiabilidad y su consistencia conceptual han permitido su adaptación y permanencia en el tiempo.

Al respecto, Gunawardane (2013) elaboró un listado de las investigaciones que han trabajado la relación entre las dimensiones de la calidad del servicio 
interno y la naturaleza del encuentro del servicio que han usado tal instrumento. Sus resultados arrojan investigaciones publicadas de 1994 a 2003, en donde se consideran las cinco dimensiones de Parasuraman et al. (1988) para medir la calidad del servicio: tangibilidad, empatía, responsabilidad, confiabilidad y seguridad, y en ocasiones se ajusta el instrumento añadiendo aspectos como adaptabilidad, espontaneidad, compromiso, confidencialidad, cortesía, competencia, imagen pública, profesionalismo, entre otros.

Es de notarse que el SERVQUAL sigue siendo útil en la medición del concepto de calidad, como en el estudio de Dedeoglu y Demirer (2015), cuyo objetivo es evaluar la percepción de la calidad del servicio entre clientes, gerentes y empleados.

Otros instrumentos utilizados en menor medida (Umamaheswari, 2014) son INTQUAL (Interal Measure of Service Qualty), desarrollado por Caruna y Pitt (1997), e INTSERVQUAL, por Frost y Kumar (2001). Estudios que aplican tales instrumentos argumentan que aunque el índice de confiabilidad es alto, la construcción y validez del SERVQUAL es mayor, por lo que es más apropiada la conceptualización y medición de la calidad del servicio interno a través de él (White y Les, 1999).

\section{Estudios de la calidad en el servicio interno por su fundamentación disciplinar y teórica}

En una segunda organización de los artículos recuperados y analizados, esquemáticamente representada en la figura 1 y que se explica a continuación, se encontraron coincidencias en la fundamentación teórica que los ubica con mayor precisión en el campo de estudios de distintas disciplinas. En primer lugar, se detectó que los estudios sobre la calidad del servicio interno se dividen en dos vertientes: los que se enfocan en la importancia del estudio para la rentabilidad económica y financiera de la empresa, a los que se denominó estudios empresariales, y los que se dirigen hacia los intereses del recurso humano, a los que se llamó estudios psicosociales.

En los estudios empresariales se encontró que las publicaciones tienen fundamento en el artículo de Heskett, Jones, Loveman, Sasser y Schlesinger (1994) de la Universidad de Harvard, en el que los autores toman a la calidad del servicio interno como enlace entre una serie de variables que resultan en ganancias y productividad para la empresa, por lo que tales estudios son abordados desde 
La calidad del servicio interno en el sector hotelero: objeto e instrumento de estudio multidisciplinar

una perspectiva estratégica económica, en la que el principal interés se ubica en la rentabilidad y el crecimiento de las organizaciones.

Por su parte, los estudios psicosociales se estructuran a partir de la importancia del recurso humano para la empresa, así, la satisfacción del trabajador surge también como interés en la investigación desde un enfoque estratégico hacia el recurso humano.

Fundamentadas en estas dos perspectivas disciplinares generales, se identificaron tres perspectivas específicas del ámbito funcional de la empresa, en las que se toma a la calidad del servicio interno como objeto e instrumento de investigación:

1. La perspectiva funcional de gestión de la calidad, basada en la filosofía y la medición de la calidad del servicio.

2. La perspectiva funcional de gestión del marketing, desde el enfoque del marketing interno para lograr la satisfacción del cliente y el cliente interno.

3. La perspectiva funcional de gestión del factor humano desde la psicología social a través del comportamiento del trabajador, para lograr la entrega exitosa del servicio.

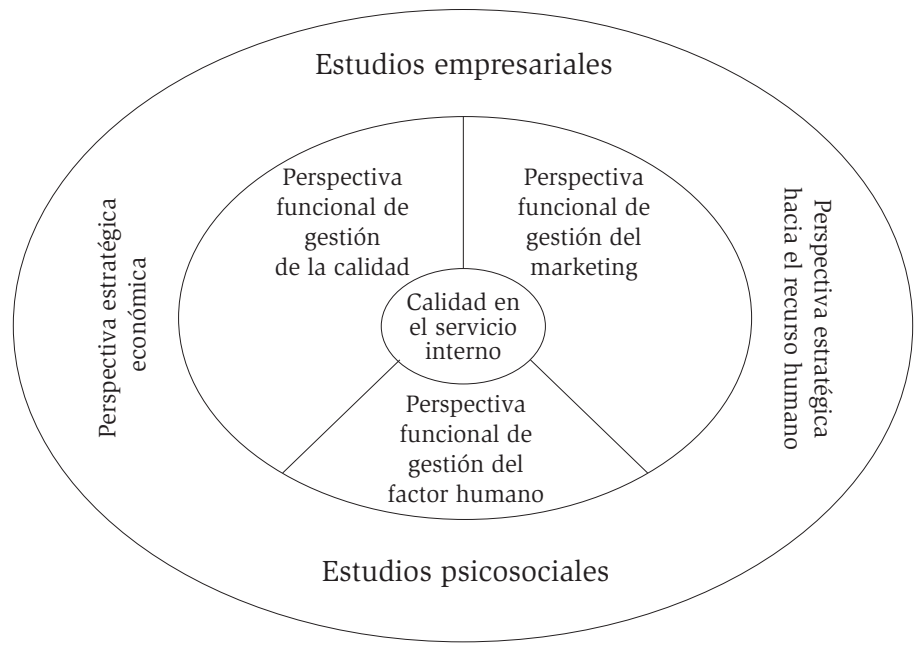

Fuente: Elaboración propia.

Figura 1. Perspectivas de los estudios empresariales y psicosociales de la calidad en el servicio interno 


\section{Estudios de la calidad en el servicio interno por sus perspectivas} estratégicas y funcionales en la organización

a) Estudios de LA CALIdAd EN El SERVICIO INTERNo DESDE LA PERSPECTIVA ESTRATÉGICA ECONÓMICA

En 1994 se identifica la aparición del término calidad del servicio interno o internal quality service en la cadena de servicio-beneficio, de Heskett et al. (1994), modelo causal enfocado al crecimiento de los ingresos y ganancias de la empresa mediante la integración de variables para el desarrollo organizacional en el contexto de la industria del servicio. Tal modelo está basado en las preposiciones de que la calidad del servicio interno conduce a empleados satisfechos, los cuales permiten la entrega de un servicio con alto valor, resultando en clientes satisfechos, provocando así su lealtad, lo que produce ganancias y crecimiento para la empresa.

En ese momento, la calidad del servicio interno en la cadena de Heskett et al. se definía como ambiente, el cual era percibido a través de los sentimientos del empleado hacia su trabajo, sus colegas y la empresa.

Con el paso del tiempo, ese modelo ha generado una serie de investigaciones, en su mayoría de orden cuantitativo, para explorar los argumentos expuestos en la cadena enfocados hacia la rentabilidad de la empresa mediante el cliente (Jones, 1997; Yee, Yeung y Chen, 2008; Chi y Gursoy, 2009; Kralj y Solnet, 2010; Pantouvakis, 2011; Yee, Yeung y Cheng, 2011), ya que los mismos autores proponen un seguimiento en la relación de variables, debido a que las relaciones expuestas son causales, por lo que se necesita una fundamentación (Yee, Yeung y Cheng, 2011).

Por lo anterior, se observa una línea de estudios sobre la apreciación del concepto de calidad del servicio interno enfocados a comprobar tanto la relación positiva directa como indirecta entre la satisfacción del cliente y la satisfacción del empleado, y su impacto en la rentabilidad de la empresa (Bernhardt, Donthu y Kennett, 2000; Harter, Schmidt y Hayes, 2002; Schneider, Macey, Barbera y Martin, 2009; Chi y Gursoy, 2009).

En cuanto a la calidad del servicio interno y la disciplina económica-administrativa, se identifica un nuevo modelo organizacional (Fadil, Singh y Joseph, 2016) denominado Innovative Internal Service Quality (IISQ), centrado en aumentar la eficiencia operacional y la satisfacción del empleado, es decir, incrementar el 
La calidad del servicio interno en el sector hotelero: objeto e instrumento de estudio multidisciplinar

desarrollo de una compañía a través de reducir costos (administrativos y suministros), generando un lugar de trabajo satisfactorio y productivo. Este modelo propone medir la influencia de la innovación organizacional (innovation leadership, managerial levers and business processes) sobre la calidad del servicio interno en las cinco dimensiones de Parasuraman et al. (1988) (reliability, responsiveness, tangibles, assurance and empathy). El trabajo se llevó a cabo en una oficina pública de Malasia.

Los estudios antes mencionados se efectúan en el campo del sector servicios, con excepción del de Pantouvakis (2011), el cual está enfocado en la industria petrolera. Dentro de la actividad turística se detectan los trabajos de Jones (1997), Bernhardt, Donthu y Kennett (2000), Chi y Gursoy (2009), y Kralj y Solnet (2010), en la industria de la hospitalidad, restaurantes de comida rápida, hoteles de cuatro estrellas y hoteles-casino, respectivamente.

Dentro de los esfuerzos para tener un equilibrio entre las ganancias de una empresa y la responsabilidad hacia sus trabajadores, se observa el concepto de responsabilidad social empresarial (RSE). Al llegar a este punto en la búsqueda de información, se notó que lograr una calidad en los servicios internos para la empresa va más allá de los esfuerzos al interior de la misma, y que es necesaria una nueva forma de organización, en donde se considere el contexto de la empresa como parte de la calidad de vida en el trabajo, el cual repercute en la satisfacción del trabajador (Mercado y García, 2007)

En esta disciplina, como en las otras, hay evidencias de que aquellas prácticas que proveen un trabajo más significativo y una alta calidad de vida en el trabajo tienen un impacto directo en las ganancias de la empresa a través del incremento de la productividad, mayores innovaciones, alta calidad y confiabilidad, así como personas más hábiles y comprometidas en todos los niveles (Palazzi y Starcher, 1997). Por lo que, por su filosofía, se considera relacionada, y en un punto de transición del concepto de calidad del servicio interno.

\section{b) Estudios de LA CALIDAD EN EL SERVICIO INTERNO DESDE LA PERSPECTIVA ESTRATÉGICA HACIA EL RECURSO HUMANO}

La perspectiva humanística de la administración, desde 1930, ya consideraba al factor humano como la esencia de una organización (Reyes, 2010); sin embargo, es de notarse que, en la búsqueda de comprender la calidad del servicio interno, se ha abierto una brecha entre los estudios que buscan probar sus 
efectos en las ganancias de la empresa, y aquellos que están más enfocados hacia la satisfacción del recurso humano, y cómo lograr ésta, para que tenga un efecto en lo anterior.

En ese sentido, se identifica el interés de la ciencia por el comportamiento del recurso humano en el interior de la organización, por lo que comprender la calidad del servicio interno ha llevado a disciplinas como la psicología social a conjugarse con la administración.

En el apartado de la perspectiva funcional de la gestión del factor humano en la organización se abordarán los estudios relacionados con esta perspectiva y la calidad del servicio interno.

\section{1) Estudios de LA CALIDAD EN EL SERVICIO INTERNO DESDE LA PERSPECTIVA FUNCIONAL DE GESTIÓN DE LA CALIDAD}

En lo que respecta al concepto de calidad del servicio, este ha sido tomado como soporte en algunas investigaciones sobre la calidad del servicio interno desde dos enfoques:

- Desde la filosofía de la calidad en el servicio, estableciendo que mejorar la calidad del servicio interno contribuye a mejorar la calidad del servicio al cliente y, por ende, el desempeño financiero de la empresa (Anderson, Fornell y Lehmann, 1994; Capon, Farley y Hoenig, 1990; Zeithaml, 2000; Rust, Moorman y Dickson, 2002).

- Midiendo la calidad del servicio interno con el modelo SERVQUAL de Parasuraman, Zeithaml y Berry (1985). Sin embargo, este es un punto de desacuerdo entre investigadores, ya que algunos (Large y König, 2009) subrayan la necesidad de un nuevo instrumento que esté ciertamente posicionado a las necesidades del cliente interno y sus expectativas, mientras que otros (Pantouvakis, 2011; Wildes, 2007) sugieren que es apropiado medir la calidad del servicio interno con las técnicas e instrumentos desarrollados en la calidad del servicio externo, tomando en consideración las diferencias entre cliente interno y externo. También hay quienes utilizan el modelo desarrollado por Caruna y Pitt (1997) denominado INTQUAL (Internal Measure of Service Quality), usando la base del SERVQUAL (Umamaheswari, 2014). 
La calidad del servicio interno en el sector hotelero: objeto e instrumento de estudio multidisciplinar

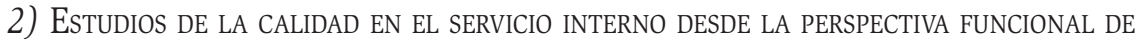
GESTIÓN DEL MARKETING.

Otra de las disciplinas en donde se identifica el término calidad del servicio interno es en el marketing, a través de sus conceptos marketing interno y calidad del servicio.

En 1976, Berry et al. introducen el concepto de marketing interno (IM), y en 1981 lo definen así: ver a los empleados como clientes internos y a los trabajos como productos que satisfacen las necesidades y deseos de los clientes mientras se dirigen hacia los objetivos de la compañía (To, Martin y Yu, 2015; Gounaris, 2006; Turkoz y Akyol, 2008).

Como en el caso de la calidad del servicio interno desde la perspectiva económica, en el marketing interno, los estudios realizados bajo este concepto tienen varias vertientes: los centrados en medir la influencia del concepto sobre la rentabilidad de la empresa (Turkoz y Akyol, 2008; Pitt y Foreman, 1999), con la satisfacción del empleado (Tag-Eldeen y El-Said, 2011; Dumitrescu, Cetina y Pentescu, 2012; Lings, 2004; Chen, Wu, Chang y Lin, 2015), y los que hacen énfasis en la medición de la satisfacción del empleado dentro del concepto marketing interno con enfoque hacia la rentabilidad de la empresa (Gounaris, 2006; Sasser y Arbeit, 1976).

Según se puede observar, el hecho de que el marketing interno defina a los empleados como clientes internos significa que se deben de tratar al interior de la empresa como clientes, incluso se menciona que crear relaciones entre empleados dentro de la organización es necesario para mejorar el desempeño de estos y de la empresa (Turkoz y Akyol, 2008), por lo que tal concepto se relaciona con el de calidad del servicio interno, ya que este también ve a los trabajadores como clientes; el punto que los une es la satisfacción del empleado al seguir predominando la filosofía de que empleados satisfechos conducen a la empresa a una mayor productividad y rentabilidad.

Desde los años ochenta, algunas investigaciones en torno a la economía de los servicios empiezan a aplicar teorías de la satisfacción del cliente y la calidad del servicio al marketing interno, y conceptos de marketing como satisfacción y calidad del servicio son usados internamente con empleados (Wildes, 2007).

Así, diversos estudios sobre marketing interno abordan conceptos relacionados con la satisfacción del cliente: el comportamiento, compromiso y actitud 
del trabajador, y la necesidad de tener empleados motivados para retenerlos como sinónimo de productividad para la empresa, pero para efectos de este trabajo, se mencionarán en la parte de calidad del servicio interno desde la perspectiva psicosocial.

De los estudios explorados, Turkoz y Akyol (2008) y Gounaris (2006) se enfocan en hoteles, mientras que el resto, a empresas de servicios.

3) Estudios de LA CALIDAD EN EL SERVICIO INTERNO DESDE LA PERSPECTIVA FUNCIONAL DE GESTIÓN DEL FACTOR HUMANO EN LA ORGANIZACIÓN.

Con la cadena de Heskett et al. en 1994, y el marketing interno de Berry et al. en 1976, se le da importancia en la investigación a la satisfacción del empleado y a las relaciones que se establecen al interior de la compañía como efecto para generar, a través del cliente, productividad y ganancias para la empresa. Sin embargo, conforme ha pasado el tiempo, se identifica en los estudios una leve tendencia de pasar de una visión de ganancias para la organización, a una mirada más humanística, y buscar el éxito o el balance entre la rentabilidad de la compañía y la satisfacción del recurso humano.

Resulta difícil separar las investigaciones en este apartado al relacionar la psicología social con el concepto calidad del servicio interno; los estudios se vuelven multidisciplinares conjugando las diferentes disciplinas revisadas anteriormente, como el estudio de To, Martin y Yu (2015), que combina, del marketing interno, la cadena de beneficio de Heskett et al., y la psicología, al abordar el compromiso y actitud del empleado en el campo de los hoteles de China; o el trabajo de Wildes (2007), que utiliza la teoría de la psicología social como base de la investigación en relación con el estereotipo del mesero, de la mercadotecnia las estrategias que vinculan la imagen percibida a la satisfacción del cliente, y de la calidad del servicio interno la literatura que ve al empleado como un valor en vez de un costo, proponiendo el manejo de la satisfacción del trabajador. Este estudio representa el más grande reto reportado por la industria de alimentos y bebidas sobre el reclutamiento y retención del personal en el puesto de meseros, y demuestra que la calidad del servicio interno atrae y retiene al personal.

Además de esos estudios multidisciplinares, también se encuentran aquellos en torno a la satisfacción del trabajador pero desde la óptica de la psicología social (Kralj y Solnet, 2010; Chow, Lai y Loi, 2014; Yee et al., 2008; Koys, 2003; Mulki, Jaramillo y Locander, 2008; Sanín y Salanova, 2014), e incluso algunos 
La calidad del servicio interno en el sector hotelero: objeto e instrumento de estudio multidisciplinar

que comprueban que el cliente percibe la satisfacción del trabajador a través de su comportamiento teniendo ampliaciones en la evaluación de la calidad del servicio (Hartline y Ferrell, 1996). En la industria hotelera, se encuentra el estudio de Karatepe y Uludag (2008), centrado en los efectos del estrés y el agotamiento sobre el desempeño del trabajo.

Durante la revisión de los artículos de esta disciplina, se observó que las variables más comunes que se abordan desde la psicología al interior de la empresa son: comportamiento del recurso humano, estrés, agotamiento, motivación, clima organizacional y liderazgo, una mezcla de enfoques entre la parte organizacional de una administración y el comportamiento del recurso humano (Boshoff y Mels, 1995).

Respecto al concepto de la calidad del servicio interno y los factores antes señalados en el área de la industria hotelera, se identifica un artículo (Chen, 2013) que trata la calidad del servicio interno desde dos factores: el liderazgo y la cultura organizacional. En él se revela que tales factores influyen en la calidad del servicio interno dentro de los hoteles de cinco estrellas.

En un estudio reciente en el campo de la industria manufacturera (Sharma, Chuen y Kingshott, 2016) se introduce el concepto de bienestar del trabajador como principal constructo en el proceso por el cual la calidad del servicio interno conduce a la satisfacción, compromiso y desempeño del trabajador, comprobando que la calidad del servicio interno tiene un efecto positivo en el bienestar del empleado, y que este modera los efectos de la satisfacción del trabajador y el compromiso sobre su desempeño.

\section{Conclusiones}

Se identifica principalmente que el término calidad del servicio interno proviene de la integración de dos nociones: calidad y servicio interno, ambas con fundamentos teóricos y metodológicos tanto en el ámbito de los estudios empresariales como en el de los estudios psicosociales. La diversidad de variables internas y externas que intervienen en su operacionalización ha provocado que su comprensión se torne compleja, lo que ha desembocado en que se convierta en objeto e instrumento de estudio multidisciplinar a través del tiempo.

Se puede afirmar que el término apareció por primera vez en la investigación de Heskett et al. (1994), y que su conceptualización se vincula con la 
filosofía de Grönroos (1982) acerca de la calidad, el marketing interno de Berry et al. (1981), y en torno al cliente interno de Stauss (1995).

La evidencia que arrojan los resultados de las investigaciones sobre la relación positiva directa entre la satisfacción del cliente y la del trabajador, y su impacto favorable en los resultados de la empresa, y que las empresas al proveer un trabajo más significativo y una alta calidad de vida laboral tienen un impacto en su productividad y rentabilidad, ha hecho que diferentes disciplinas se conjuguen en el estudio de la calidad del servicio interno, abordándolo desde dos perspectivas generales: la perspectiva estratégica económica y la perspectiva estratégica del recurso humano, y tres perspectivas funcionales: la gestión de la calidad, la gestión del marketing y la gestión del factor humano.

Cabe notar que, como instrumento de investigación, el concepto de calidad del servicio interno se evalúa principalmente por medio de la herramienta SERVQUAL de Parasuraman et al. (1988); dada su construcción y validez, se utiliza en su mayoría en investigaciones de orden cuantitativo.

Es importante señalar que los estudios sobre la calidad del servicio interno no solo aplican para empresas de servicios, ya que el servicio entre empleados se da tanto en esas empresas como en las que fabrican bienes. Para efectos del interés de esta investigación, se concluye que en el campo del sector hotelero se detectaron cinco estudios: dos desde la perspectiva de la gestión económica, dos desde la gestión funcional del marketing y uno desde la perspectiva funcional del factor humano.

En cuanto a la orientación de los estudios, se observa una leve tendencia en ellos de pasar de un enfoque económico a uno más humanístico de responsabilidad social, y aunque sigue habiendo algunos centrados en la perspectiva económica, estos van de la mano de la satisfacción del trabajador y la generación de un lugar de trabajo satisfactorio que impacte de forma positiva en su bienestar.

Por último, se detecta una necesidad en la investigación de este concepto hacia los estudios cualitativos, ya que los artículos sugieren se indaguen variables demográficas como edad, género, nivel de educación; variables psicográficas como búsqueda al cambio y toma de decisiones, y variables económicas como salarios, bonos y otros beneficios, en relación con la calidad del servicio interno, mediante herramientas como la entrevista, a fin de ahondar en estos factores. Así pues, se sugiere que la línea de investigación que ahora se aborde sobre este concepto sea desde un aspecto social, en donde se analicen estas variables. 
La calidad del servicio interno en el sector hotelero: objeto e instrumento de estudio multidisciplinar

\section{Fuentes consultadas}

Aguilera, R. V., Rupp, D. E., Williams, C. A. y Ganapathi, J. (2007). Putting the $\mathrm{S}$ back in corporate social responsibility: A multilevel theory of social change in organizations. Academy of Management Review, 32(3), 836863. Recuperado de http://doi.org/10.5465/AMR.2007.25275678

Anderson, E. W., Fornell, C. y Lehmann, D. R. (1994). Customer satisfaction, market share and profitability: Findings from Sweden. Journal of Marketing, 58(3), 53-66.

Bernhardt, K., Donthu, N. y Kennett, P. (2000). A Longitudinal analysis of satisfaction and profitability. Journal of Business Reseacrh, 47(2), 161-171.

Boshoff, Ch. y Mels, G. (1995). A causal model to evaluate the relationships among supervision, role stress, organizational commitment and internal service quality. European Journal of Marketing, 29(2), 23-42.

Capon, N., Farley, J. y Hoenig, S. (1990). Determinants of financial performance: A meta-analysis. Management Science, 36(10), 1143-1159.

Caruna, A. y Pitt, L. (1997). An internal measure of service quality and the link between service quality and business performance. European Journal of Marketing, 31(8), 604-616.

Chen, S., Wu, W., Chang, Ch. y Lin, Ch. (2015). Job rotation and internal marketing for increased job satisfaction and organizational commitment in hospital nursing staff. Journal of Nursing Management, 23, 297-306.

Chen, W.-J. (2013). Factors influencing internal service quality at international tourist hotel. International Journal of Hospitality Management, 35, $152-160$.

Chi, Ch. y Gursoy, D. (2009). Employee satisfaction, customer satisfaction, and financial performance: An empirical examination. International Journal of Hospitality Management, 28, 245-253.

Chow, Ch., Lai, J. y Loi, R. (2014). Motivation of travel agents' customer service behavior and organizational citizenship behavior: The role of leadermember Exchange and internal marketing orientation. Tourism Management, 48, 362-369.

Cook, S. (2004). Measuring Customer Service Effectiveness. Burlington: Gower Publishing Company.

Dedeoglu, B. y Demirer, H. (2015). Differences in service quality perceptions of stakeholders in the hotel industry. International Journal of Contemporary Hospitality Management, 27(1), 130-146. 
Dumitrescu, L., Cetina, I. y Pentescu, A. (2012). Employee satisfaction measurement - Part of internal marketing. Review of International Comparative Management, 13(1), 37-48.

Fadil, H., Singh, K. y Joseph, C. (2016). Influence of organizational innovation towards internal service quality in мвкs. Procedia-Social and Behavioral Sciences, 224, 317-324.

Finn, D., Baker, J., Marshall, G. y Anderson, R. (1996). Total quality management and internal customers: Measuring internal service quality. Journal of Marketing Theory and Practice, Summer, 35-50.

Freeman, R. E., Wicks, A. C. y Parmar, B. (2004). Stakeholder theory and "The corporate objective revisited”. Organization Science, 15(3), 364-369. Recuperado de http://doi.org/10.1287/orsc.1040.0066

Friedman, M. (1970). The social responsibility of business is to increase its profits. The New York Times Magazine, 6. Recuperado de http://doi. org/10.1007/978-3-540-70818-6_14

Frost, F. y Kumar, M. (2001). Service quality between internal customer and internal suppliers in an international airline. International Journal of Quality \& Reliability Management, 18, 371-386.

Gounaris, S. (2006). Internal-Market orientation and its measurement. Journal of Business Research, 59, 432-448.

Grönroos, Ch. (1982). An applied service marketing theory. European Journal of Marketing, 16(7), 30-41.

Gunawardane, G. (2009). Relationship between dimensions of internal service quality and the nature of the internal service encounter - A study in the healthcare industry. California Journal of Operations Management, $7(1), 21-30$.

Hallowell, R., Schlesinger, L. y Zornitsky, J. (1996). Internal service quality, customer and job satisfaction: Linkages and implications for managers. Human Resource Planning, 19, 20-31.

Hart, S. L. (1997). Beyond greening: Strategies for a sustainable world. Harvard Business Review, 66(11), 1-10.

Harter, J., Schmidt, F. y Hayes, T. (2002). Business-Unit level relationship between employee satisfaction, employee engagement, and business outcomes: A meta-analysis. Journal of Applied Psychology, 87(2), 268269.

Hartline, M. y Ferrell, O. (1996). The management of customer-contact service employees: An empirical investigation. Journal of Marketing, 60, 52-70. 
La calidad del servicio interno en el sector hotelero: objeto e instrumento de estudio multidisciplinar

Heskett, J., Jones, T., Loveman, G., Sasser, W. y Schlesinger, L. (1994). Putting the service-profit chain to work. Harvard Business Review, MarchApril, 164-170.

Homburg, C. y Stock, R. (2005). Exploring the conditions under which salesperson work satisfaction can lead to customer satisfaction. Psychol. Market., 22(5), 393-420.

Jones, P. (1997). The hospitality service profit chain: An effective research design. Hospitality Management, 16, 289-296.

Jun, M. y Cai, S. (2010). Examining the relationships between internal service quality and its dimensions, and internal customer satisfaction. Total Quality Management, 21(2), 205-223.

Karatepe, O. y Uludag, O. (2008). Role stress, burnout and their effects on frontline hotel employees' job performance: Evidence from Northern Cyprus. International Journal of Tourism Research, 10, 111-126.

Kasim, A. (2006). The need for business environmental and social responsibility in the tourism industry. International Journal of Hospitality \& Tourism Administration, 7(1), 1-22. Recuperado de http://doi.org/10.1300/ J149v07n01_01

Koys, D. (2003). How the achievement of human-resources goals drives restaurant performance. The Cornell Hotel and Restaurant Administration Quarterly, 44(1), 1-96.

Kralj, A. y Solnet, D. (2010). Service climate and customer satisfaction in a casino hotel: An exploratory case study. International Journal of Hospitality Management, 29, 711-719.

Large, R. O. y König, T. (2009). A gap model of purchasing's internal service quality: Concept, case study and internal survey. Journal of Purchasing \& Supply Management, 15, 24-32.

Lings, I. (2004). Internal market orientation construct and consequences. Journal of Business Research, 57, 405-413.

Mehrparvar, E., Shahin, A. y Shirouyehzad, H. (2012). Prioritizing internal quality dimensions using TOPSIS Technique (With a case study in Isfahan Steel Mill Co.). International Journal of Business and Social Science, 3(2), 210-217.

Mercado, P. y García, P. (2007). La responsabilidad social en empresas del Valle de Toluca (México). Un estudio exploratorio. Estudios Gerenciales, 23(102), 119-135. 
Mulki, J., Jaramillo, J. y Locander, W. (2008). Effect of ethical climate on turnover intention: Linking atitudinal-and stress theory. Journal of Business Ethics, 78, 559-574.

Nazeer, S., Zahid, M. y Azeem, M. (2014). Internal service quality and job performance: Does job satisfaction mediate? Journal of Human Resources Management and Labor Studies, 2(1), 41-65.

Palazzi, M. y Starcher, G. (1997). Corporate Social Responsibility and Business Sucess. The European Baha'i Business Forum. Recuperado de http:// centreonphilanthropy.com/files/kb_articles/1269270655EBBF $\% 2520$ Corporate \% 2520Responsibility \% 2520and \% 2520Business \% 2520Succe ss.pdf $[2015,11$ de noviembre].

Pantouvakis, A. (2011). Internal service quality and job satisfaction synergies for performance improvement: Some evidence from a B2B environment. Journal of Targeting, Measurement and Analysis for Marketing, $19,11-22$.

Parasuraman, A., Zeithaml, V. y Berry, L. (1985). A conceptual model of service quality and its implications for future research. Journal of Marketing, 49, 41-50.

Parasuraman, A., Zeithaml, V. y Berry, L. (1988). SERVQUAL: A multiple-item scale for measuring consumer perceptions of service quality. Journal of Retailing, 64(1), 12-40.

Pitt, L. y Foreman, S. (1999). Internal marketing role in organizations: A transaction cost perspective. Journal of Business Research, 44, 25-36.

Reyes, A. (2010). Administración moderna. México: Limusa.

Rust, R., Moorman, Ch. y Dickson, P. (2002). Getting return in quality: Revenue expansion, cost reduction, or both? Journal Marketing, 66, 7-24.

Sanín, J. A. y Salanova, M. (2014). Satisfacción laboral, el camino entre el crecimiento psicológico y el desempeño laboral en empresas colombianas industriales y de servicios. Universitas Psychologica, 13(1), 1-22. doi:10.11144/Javeriana.UPSY13.slep

Sasser, E. y Arbeit, S. (1976). Selling jobs in the service sector. Business Horizons, June, 61-65.

Schneider, B., Macey, W., Barbera, K. y Martin, N. (2009). Driving customer satisfaction and financial success thought employee engagement. People \& Strategy, 32(2), 22-27.

Seibert, J. y Lingle, J. (2007). Internal customer service: Has it improved? Quality Progress, March, 35-40. 
La calidad del servicio interno en el sector hotelero: objeto e instrumento de estudio multidisciplinar

Sharma, P., Chuen, T. y Kingshott, R. (2016). Internal service quality as a driver of employee satisfaction, commitment and performance - Exploring the focal role of employee well-being. Journal of Service Management, 27(5), 1-43.

Stauss, B. (1995). Internal services: classification and quality management. Journal of Service Industry Management, 6(2), 62-78.

Tag-Eldeen, A. y El-Said, A. (2011). Implementation of internal marketing on a simple of Egyptian five-star hotels. Anatolia: An International Journal of Tourism and Hospitality Research, 22(2), 153-167.

To, W., Martin, E. y Yu, B. (2015). Effect of management commitment to internal marketing on employee work attitude. International Journal of Hospitality Management, 45, 14-21.

Turkoz, I. y Akyol, A. (2008). Internal marketing and hotel performance. Anatolia: An International Journal of Tourism and Hospitality Research, 19, 149-177.

Umamaheswari, J. (2014). Exploring internal service quality in a manufacturing organization-A study in Lucus TVS, Chennai. Procedia Economics and Finance, 11, 710-725.

Vanniarajan, T. y Subbash, B. (2011). Internal service quality and its consequences in commercial banks: A HR perspective. Global Management Review, 6, 42-57.

White, Ch. y Les, R. (1999). INTERSERVQUAL: An investigation of the dimensions and measurement of internal service quality in the hospitality industry. Australian Journal of Hospitality Management, 6(2), 13-22.

Wildes, V. (2007). Attracting and retaining food servers: How internal service quality moderates occupational stigma. Hospitality Management, 26, 4-19.

Yee, R., Yeung, A. y Chen, E. (2008). The impact of employee satisfaction on quality and profitability in high-contact service industries. Journal of Operations Management, 26, 651-668.

Yee, R., Yeung, A. y Cheng, T. (2011). The service-profit chain: An empirical analysis in high contact service industries. Int J. Production Economics, 130, 236-245.

Zeithaml, V. (2000). Service quality, and the economic worth of customers: What we know and what we need to learn. Journal of the Academy of Marketing Science, 28(1), 67-85. doi:10.1177/0092070300281007 